\title{
ENERGETICS, BONDING MECHANISM AND ELECTRONIC STRUCTURE OF CERAMIC/CERAMIC AND METAL/CERAMIC INTERFACES
}

\author{
An Annual Progress Report for
}

DOE Grant No. DE-FG02-88ER45372-6

Submitted to the

\section{U.S. DEPARTMENT OF ENERGY}

by

\author{
A.J. Freeman, Principal Investigator \\ Department of Physics and Astronomy \\ Northwestem University \\ Evanston, IL 60208-3112 \\ Tel. 708-491-3343; Fax 708-491-5082 \\ Email: art@freeman.phys.nwu.edu
}

\section{Period of Performance: April 1, 1993 to March 31, 1994 DISCLAIMER}

\footnotetext{
This report was prepared as an account of work sponsored by an agency of the United States Government. Neither the United States Government nor any agency thereof, nor any of their employees, makes any warranty, express or implied, or assumes any legal liability or responsibility for the accuracy, completeness, or usefulness of any information, apparatus, product, or

- process disclosed, or represents that its use would not infringe privately owned rights. Refer-

$=\quad$ ence herein to any specific commercial product, process, or service by trade name, trademark,

* manufacturer, or otherwise does not necessarily constitute or imply its endorsement, recommendation, or favoring by the United States Government or any agency thereof. The views and opinions of authors expressed herein do not necessarily state or reflect those of the United States Government or any agency thereof.
}

This report was prepared as an account of work sponsored by the United States Government. Neither the United States nor the Department of Energy, nor any of their employees, nor any of their contractors, subcontractors, or their employees, makes any warranty, express or implied, or assumes any legal liability or responsibility for the accuracy, completeness, or usefulness of any information, apparatus, product or process discosed or represents that its use would not infringe privately-owned rights. 


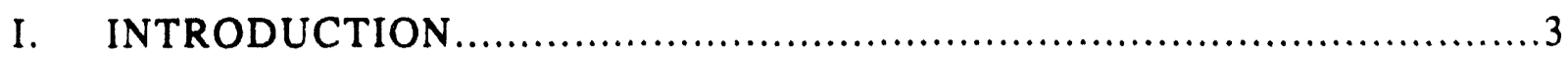

A. Background Perspective...........................................................

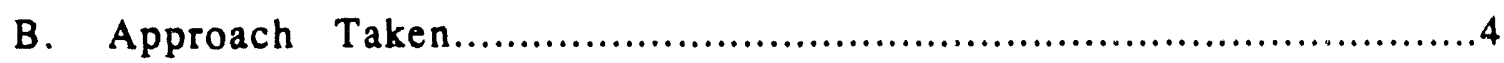

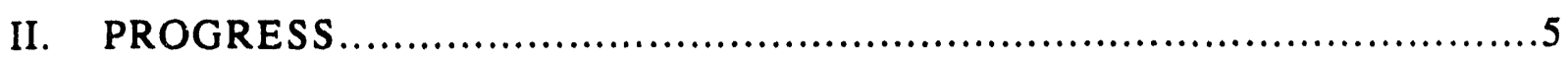

A. Metal/Ceramic Interfaces Studies......................................................5

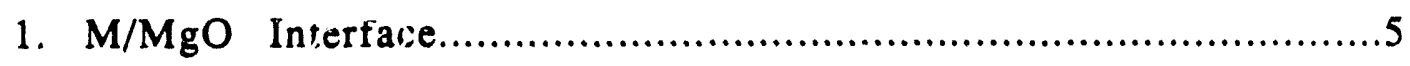

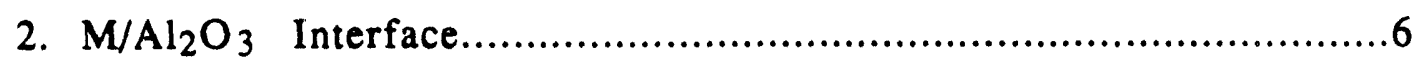

B. Optimized Geometry, Static Potential and Ferroelectric Instability in PbT.............8

C. $\mathrm{VO}_{2}$ and $\mathrm{TiO}_{2}$ Surfaces, Interfaces and Superlattices....................................10

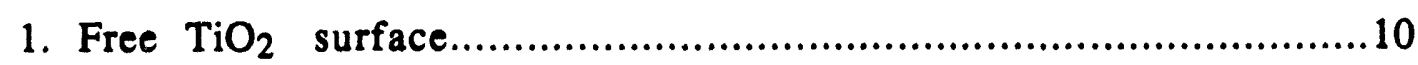

2. $\mathrm{VO}_{2}$ and $\mathrm{TiO}_{2}$ thin films and multilayers ........................................13

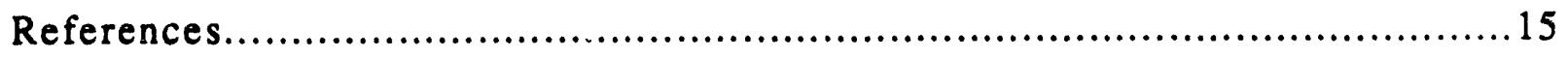

Publications of P.I. supported by DOE (1990-present) ..........................................17

Reprint priprixts - removed

$$
\because
$$




\section{INTRODUCTION}

\section{A. Backgroned Perspective}

This DOE project has several main thrusts. It had as its first objective, the theoretical study of the metal/ceramic interface in close collaboration with experimentalists at Argonne National Laboratory (K. Merkle, et al.) and at Northwestern University (D. Seidman and his group) and to take some first steps in gaining a basic understanding of a number of key issues involved in determining the structure and properties of heterophase ceramic interfaces. It was widely recognized that some of the most important chemical and physical properties of materials in high technology applications are either controlled or are strongly influenced by the presence and properties of interfaces. As a result, this field attracted growing experimental and theoretical efforts. While a good deal of attention had been given to the study of the macroscopic properties of metal/ceramic interfaces, very little was known about the atomic structure and composition of interfaces and their relation to interface properties. Thus, we set out to address theoretically some of these fundamentional issues at the atomic level using our highly precise local density total energy full potential linearized augmented plane wave (FLAPW) method - which we had successfully applied to the study of the cuprate high temperature superconductors. In this first, still ongoing, phase of the project, we hoped to provide insight into the energetics and nature of bonding, the bonding mechanism, and structure of metal/ceramic interfaces, notably metal/MgO and $\mathrm{Al}_{2} \mathrm{O}_{3}$ interfaces. Specific questions addressed included the range of metallic bonding from the interface and the nature and degree of valence changes (charge transfer), anisotropy of the bonding of the interface, and in the case of $\mathrm{Fe} / \mathrm{MgO}$, magnetism in a $2 \mathrm{D}$ environment.

In the second thrust of our project, we worked closely with D. Lam and his group at ANL on the emergingly important ferroelectronic materials and on studying theoretically their epitaxially grown oxide thin films fabricated as a superlattice structure.[1] This group has focussed on the controlled growth of epitaxial films of multicomponent oxides so that artificial materials or structures in multilayer form can be fabricated where new and interesting physical properties may be created. Oxide materials have a wealth of unique and interesting physical properties that can be used for vajous rechnological applications. Many oxide materials have been extensively used in the form of thin films because the applications involve microdevices which require the materials to be fabricald into micron or submicron scales. As emphasized by the ANL group, oxides form with all metallic elements of the periodic table; therefore, a much greater selection of compounds and crystallographic possibilities is available. The variety of available compounds multiplies rapidly when one includes multicomponent oxides. The range of physical properties is comparably varied. Thus, as they have demonstrated in a number of cases, one can choose candidate substrate and epitaxial overlayer materials that meet the requirements of minimum interface strain while simultaneously selecting materials with varied or contrasting physical properties. The possibility 
for epitaxial fabrication of multilayer structures with individually tailored physical properties in each of the layers is almost unlimited.

In collaboration with Lam's group, we have studied theoretically[2] (i) a model $\mathrm{VO}_{2} / \mathrm{TO}_{2}$ superlattice, (ii) the bulk and surface properties of $\mathrm{VO}_{2}$ and $\mathrm{TiO}_{2}$, and (iii) bulk $\mathrm{PbTiO}_{3}, \mathrm{PbZrO}_{3}$, $\mathrm{BaTiO}_{3}$ and $\mathrm{SrTiO}_{3}$. Specifically, since Lam, et al., successfully synthesized $\mathrm{VO}_{2} / \mathrm{TiO}_{2}$ multilayers, we have carried out some computationally complex full potential linear muffin-tin orbital (FLMTO) electronic structure calculations of model $\mathrm{VO}_{2} / \mathrm{TiO}_{2}$ superlattices. Films of $\mathrm{TiO}_{2}$ and $\mathrm{VO}_{2}$ have been grown epitaxially by the MOCVD technique and studied in detail by Lam et al. Hence, as part of these studies, we have investigated in detail, also by the FLMTO method, the properties of bulk and thin film $\mathrm{TiO}_{2}$ and $\mathrm{VO}_{2}$. Simultaneously, we have obtained a highly realistic FLAPW simulation of a $\mathrm{TiO}_{2}$ surface - which is of special interest in surface physics and electrochemistry because of its unusual reactive properties.

Also in collaboration with the same ANL group, we have been studying ferroelectronic perovskites, notably, $\mathrm{PbTiO}_{3}, \mathrm{BaTiO}_{3}, \mathrm{SrTiO}_{3}$ and $\mathrm{PbZrO}_{3}$. To this end, frozen phonon total energy calculations were employed to study the origin of the ferroelectric transition in $\mathrm{PbTiO}_{3}$. Comparison studies with tetragonal $\mathrm{BaTiO}_{3}$ to which it is isomorphic, were performed in order to confirm and understand the occurrence of a ferroelectric instability in the tetragonal structure of $\mathrm{PbTiO}_{3}$. These simulations and those for $\mathrm{SrTiO}_{3}$ and $\mathrm{PbZrO}_{3}$ put us in good position to try to understand the origin of the antiferroelectric transitions in $\mathrm{PbZrO}_{3}$ which is similar in its electronic and structural character.

The studies underway on the perovskite titanitates will permit us to work closely with the new ANL Interface Materials Group (now headed by Dieter Wolf) on multicomponent oxides such as $\mathrm{PbTiO}_{3}, \mathrm{BaTiO}_{3}$ and $\mathrm{PbZrO}_{3}$ as this offers the possibility of producing materials with unusual properties by combining different ferroelectric, antiferroelectric and electro-optic materials in multilayer and superlattice configurations. The goal of the ANL synthesis/characterization effort is to produce materials with new electrical, optical and mochanical properties by combining different ferroelectric, antiferroelectric, and electro-optic materials in multilayer and superlattice configuraibas. The advent and availability of the Cray C-90 gives us the power (speed and memory) treating these complex systems.

\section{B. Approach Taken}

In our work, state-of-the-art local density electronic structure methods are developed and employed to study, by means of computer simulation, the problems addressed. The methods include the all-electron total energy FLAPW (full potential linearized augmented plane wave) method which has been demonstrated to be the most highly precise approach to the study of bulk solids, surfaces, interfaces and superlattices.[3] In the FLAPW method, no shape approximations 
are made to either the charge density or the potential. We have recently added the means to calculate atomic forces needed for the determination of site preference, lattice relaxation, phonon frequencies, soft modes, etc. We have also adapted the FLMTO (full potential linearized muffin-tin orbital) method[4] to the study of superlattices. Since this approach is significantly faster, in most instances, than the FLAPW method, it can be employed to treat larger supercells in the simulations of multilayer systems.

\section{PROGRESS}

\section{A. Metal/Ceramic Interface Studies}

1. MMgO Interface. We obtained, for the first time, a detailed understanding of the nature of the metal/MgO interface.[5] It was shown by means of total energy slab calculations that $\mathrm{Ag}$ (and later $\mathrm{Fe}$ and $\mathrm{Co}$ ) atoms are preferentially absorbed above the $\mathrm{O}$ atoms on the $\mathrm{MgO}(001)$ surface. (Note that in model calculations that include the image and repulsion terms, the noble metal adatoms tend toward the site above the positive ion in alkali halides.) The present result indicates the important role of the weak oxygen-metal hybridization in determining the site preference. Compared with the clean $\mathrm{MgO}$ surface, there is only a very small decrease $(<0.02$ electrons) of the total charge on the $\mathrm{Mg}$ and $\mathrm{O}$ atoms. Whereas the interface $\mathrm{O}$ atom is slightly metallized, the $\mathrm{Mg}$ is less influenced by the $\mathrm{Ag}$ overlayer because the $\mathrm{Mg}$ valence states are well above $E_{F}$. Recently, we extended the same procedure to study another important interface, namely $\mathrm{Ag} / \mathrm{CdO}(001)$. Total energy results indicate that $\mathrm{Ag}$ adatoms also prefer to lie on top of $\mathrm{O}$, not on $\mathrm{Cd}$. However, since the electro-negativities of $\mathrm{Ag}$ and $\mathrm{Cd}$ are so close (1.90 vs. 1.63), Ag-O interactions in $\mathrm{Ag} / \mathrm{CdO}(001)$ are expected to be considerably stronger than in $\mathrm{Ag} / \mathrm{MgO}(001)$. In fact, total energy calculations show that the equilibrium $\mathrm{Ag}-\mathrm{O}$ distance in $\mathrm{Ag} / \mathrm{CdO}(001)$ is 4.1 a.u., which is much smaller than the corresponding value of 5.1 a.u. in $\mathrm{Ag} / \mathrm{MgO}(001)$. Calculations for the cohesion energy, which is a direct measure of the bond strength, are in progress.

These results were so dramatic that we carried out a similar study of $\mathrm{Fe} / \mathrm{MgO}(001)$ and then Co/MgO(001) - in large part because of the interest of $M$. Brodsky at ANL in our seeking a benign substrate fis 2D magnetic experiments. We found[6], surprisingly, that the calculated magnetic

moment $1.07 \mu_{\mathrm{B}}$, is very close in value to that of a free-standing Fe monolayer and hence larger th thut of the bcc Fe free surface value $\left(2.98 \mu_{B}\right)$ or of Fe monolayers on noble metal surfaces (2.98 $\mu_{\mathrm{B}}$ on $\mathrm{Au}$ and $\left.\mathrm{Ag}(001)\right)$. For all metal overlayers, the weak hybridization and negligible charge transfer causes almost ideal 2D magnetism. Very recently, we investigated the effects of a protective gold overlayer on the $\mathrm{Fe} / \mathrm{MgO}(001)$ system which was later investigated by Fulcher, et al.[7] at ANL. The Fe magnetic moment was found to remain as large as $2.95 \mu_{\mathrm{B}}$, close to the value of $2.98 \mu_{\mathrm{B}}$ in $\mathrm{Fe} / \mathrm{Au}(001)$. Calculated charge and spin densities for $\mathrm{Au} / \mathrm{Fe} / \mathrm{Mg}(001)$ 
are shown in Fig. 1. Again, this indicates the extremely weak effects of the $\mathrm{MgO}$ substrate on the Fe magnetization even when covered with $\mathrm{Au}$.

In order to explore the possibility of growing magnetic thin films on $\mathrm{MgO}$ from "nonmagnetic" materials, we also calculated $\mathrm{Pd} / \mathrm{Mg}(001)$ and $\mathrm{Rh} / \mathrm{MgO}(001)$.[8] Surprisingly, while its magnetic moment is $0.35 \mu_{\mathrm{B}}$ in the free standing monolayer case, the Pd layer becomes magnetically dead on $\mathrm{MgO}(100)$ - suggesting the sensitivity of the $4 \mathrm{~d}$ magnetism of $\mathrm{Pd}$ to environmental changes. By contrast, the magnetic moment of $\mathrm{Rh} / \mathrm{MgO}(001)$ is as large as $1.4 \mu_{\mathrm{B}}$, which is only $4 \%$ smaller than that for the free $\mathrm{Rh}$ monolayer. This value is considerably larger than that for $\mathrm{Rh} / \mathrm{Ag}(001)$, i.e. $0.95 \mu_{\mathrm{B}}$, suggesting the strong possibility of realizing $4 \mathrm{~d}(\mathrm{Rh})$ magnetism on the $\mathrm{MgO}(001)$ substrate. To see whether this magnetism will be diminished by a protective $\mathrm{Ag}$ or $\mathrm{Au}$ coating layer, we will investigate the $\mathrm{Ag}(\mathrm{Au} / \mathrm{Rh} / \mathrm{MgO}(001)$ system. Experiments on this system to check our predictions will be undertaken by Sam Bader and his group at ANL.

Earlier, the somewhat startling results for $\mathrm{Fe} / \mathrm{MgO}$ had a significant effect on Sam Bader's group at MSD, ANL which led them to undertake and perform some magnetic studies also involving neutron studies by Felcher, et al.[7] at the IPNS. These results for the Au covered $\mathrm{Fe} / \mathrm{Mg}(001)$ system at room temperature did not show enhanced magnetism for thick Fe layers at room temperature. More recently, Jang, Seidman, and Merkle[9] appear to have confirmed our predictions, by means of HREM and APFMM measurements that in the case of $\mathrm{Cu} / \mathrm{MgO}$, the $\mathrm{Cu}$ atom is adsorbed over the $O$ site. This was the first demonstration that by combining these microscopies, it is possible to determine the chemical identity of the terminating plane on the oxide side of a Cu/MgO(111)-type interface directly without any deconvolution of the experimental data.

2. $\mathrm{M} / \mathrm{Al}_{2} \mathrm{O}_{3}$ Interface. $\mathrm{Pd} / \mathrm{Al}_{2} \mathrm{O}_{3}$ interfaces were labelled by Eastman and Rühle[10] as being "useful as a starting point to study model systems". Our studies demonstrated that the presence of interface states enhances the interface Pd density of states (DOS), that the interface oxygen layer has a sharp peak in the DOS near $E_{F}$ and that the main band produced by hybridization between $\mathrm{Al}$ and $\mathrm{O}$ is narrower than that of the bulk because of the small $\mathrm{Al}$ coordinati number.[11] The sharp interface states show that the interaction between $\mathrm{Pd} / \mathrm{O}$ is nearly ionis and that the location of the states at $E_{F}$ causes the instability of this interface. From these results, we were able to predict that a stable $\mathrm{Pd} / \mathrm{Al}_{2} \mathrm{O}_{3}$ interface can be obtained by changing the oxygen number in the interface layer or the Pd stacking because the variation of the number of cations moves the Fermi level and the different coordination of $\mathrm{Pd}$ and $\mathrm{O}$ causes the variation of the interface states.

As part of these studies on the electronic structure of the noble metal oxides, we have 

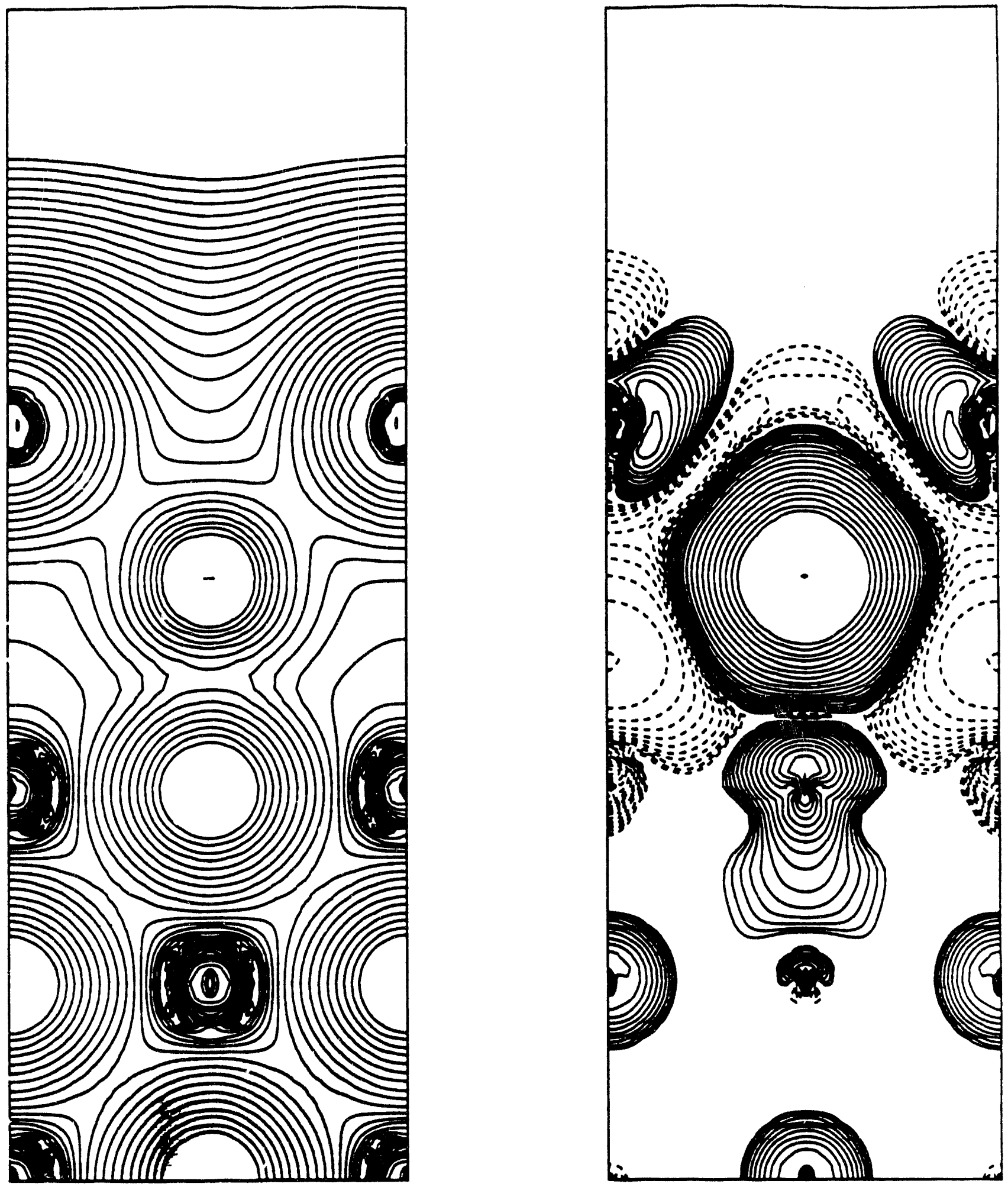

Figure 1. The calculated (a) charge and (b) spin densities of $\mathrm{Ag} / \mathrm{Fe} / \mathrm{MgO}(001)$. Contours shown on the vertical (010) plane start ${ }^{-0}$ from $\pm / 1 \times 10^{-4}$ e/a.u. ${ }^{3}$ and increase successively by a factor of $\sqrt{2}$. Dashed lined in panel b represents negative spin density. 
performed band structure calculations for PdO, PtO and AgO using the local density FLAPW method.[12] The calculated band structures show that $\mathrm{Pd}$ and $\mathrm{Pt}$ atoms are strongly hybridized with oxygen atoms in comparison with $3 \mathrm{~d}$ oxides and that their oxides show metallic behavior. The tetragonal field splitting of the $d$ levels is not sufficient to obtain a gap. However, the calculated DOS are well matched with the peaks of XPS or UPS experiments. Even though local density approximation (LDA) calculations do not give band gaps in $\mathrm{PdO}$ and $\mathrm{PtO}$, their band gaps are of the band type induced by the crystal field, rather than the Mott-Hubbard type or charge transfer type like the $3 \mathrm{~d}$ oxides. In AgO, the monoclinic crystal field splitting of the $\mathrm{d}$ states induces a small indirect band gap of $0.03 \mathrm{eV}$. This splitting leads to valences of $\mathrm{Ag}$ that have $\mathrm{Ag}^{+1}$ and $\mathrm{Ag}^{+2}$ configurations rather than $\mathrm{Ag}^{+1}$ and $\mathrm{Ag}^{+3}$, since the small $\mathrm{p}$-d energy separation prefers the existence of oxygen holes.

\section{B. Optimized Geometry, Static Potential and Ferroelectric Instability in $\mathrm{PbTiO}_{3}$}

As part of our studies of the perovskite ferroelectrics, we have carried out extensive studies on $\mathrm{PbTiO}_{3}$. A detailed preprint[13] is given as Appendix A to this proposal - which also includes full references. As stated there, it is well known that $\mathrm{PbTiO}_{3}$ is one of the perovskite ferroelectrics which have been employed extensively. Solid state applications include switching devices and a large variety of signal processing devices. It undergoes a phase transition at $763 \mathrm{~K}$ from cubic perovskite to a tetragonal ferroelectric phase isomorphic with tetragonal $\mathrm{BaTiO}_{3}$. There are some differences however between these two ferroelectrics. For $\mathrm{PbTiO}_{3}$, the tetragonal phase persists even at low temperature, while $\mathrm{BaTiO}_{3}$ undergoes several structural transitions in this temperature range; further, its c/a ratio and ferroelectric displacements are larger than those for $\mathrm{BaTiO}_{3}$, leading $\mathrm{PbTiO}_{3}$ to have higher values of the Curie temperature and spontaneous polarization.

Despite its scientific and technological importance, the electronic properties and the basic mechanism of the ferroelectric phase transition in $\mathrm{PbTiO}_{3}$ have not yet been fully elucidated. There are many difficulties in investigating the phase transition properties experimentally and in interpretin ghe observations. Theoretical calculations concerning the dynamical properties, such as phonom and phase transitions, have employed empirical, semi-empirical methods, model calculations and very recently, first-principles calculations by the FLAPW method.[3]

Most first-principles calculations of the electronic and dynamical properties of ferroelectrics (not only $\mathrm{PbTiO}_{3}$ but $\mathrm{BaTiO}_{3}, \mathrm{KNbO}_{3}$, etc.) have been carried out with just the experimental structural parameters. This restricts the possibilities of these methods to simulate and predict changes of the electronic structure and properties under different conditions. Only recently have calculations of the dynamical properties with optimized displacement geometry and simulations of the $\mathrm{KNbO}_{3}$ ferroelectric ground state been done as a result of the development of the fast and 
sufficiently frecise FLMTO method.[4] Obviously, theoretical methods which are both precise enough to calculate the optimized geometry for ferroelectric displacements and able to perform extensive total energy calculations are required because of the considerable sensitivity of ferroelectrics to defects, impurities and pressure.

We have performed first-principles calculations of the ferroelectric state structurai pisameters, namely c/a, ferroelectric displacements and volume, for $\mathrm{PbTiO}_{3}$ [see Appendix A]. We used results of previous FLAPW calculations $[14,15]$ obtained at the experimental c/a and volume as benchmarks and performed extensive calculations of the total energy as a function of the entire parameter space by the FLMTO method. Thus we determined: (i) the optimized structure of ferroelectric ground state; (ii) parameters of the static potential for the equilibrium cubic and tetragonal phases; and (iii) the ratio of the soft mode frequencies for these phases in order to gain insight into the peculiarities of phase transitions at finite temperature using results of model calculations $[16,17]$. The calculated structural parameters for the ferroelectric ground state are found to be in good agreement with experiment.

The different mechanisms (displacive or order-disorder) of phase transitions for such materials as bulk $\mathrm{PbTiO}_{3}, \mathrm{SrTiO}_{3}$ and $\mathrm{BaTiO}_{3}$ have been a subject of both theoretical and experimental investigations. However, the nature of this transition in even such a textbook material as $\mathrm{PbTiO}_{3}$ is still unclear. The delicate balance of long-range Coulomb and short-range repulsive interactions control the local distortion and hence the process driving the phase transformation; this balance should be taken into account along with the effects of thermal vibrations.

Now, the $\Phi^{4}$ model potential is widely employed for modelling systems that undergo displacive and order-disorder phase transitions. The Hamiltonian of the so-called $\Phi^{4}$ model, has been described elsewhere $[18,19,16,17]$ and is presented and discussed in Sec. III. Direct molecular dynamic simulations with the $\Phi^{4}$ Hamiltonian have shown that the crossover between displacive and order disorder type phase transitions is controlled by the static potential of the $\Phi^{4}$ model, namely it is controlled by the shape and depth of the potential well [17].

Estimates of the $\Phi^{4}$ static potential parameters for equilibrium cubic and tetragonal phases have been obtained by FLMTO total energy results fit in the form

$$
\mathrm{E}=a u^{2}+b u^{4}
$$

where $u$ denotes soft mode displacements in dimensionless units. Our results for the $a$ and $b$ parameters and for the depth of the double well potential indicate that this set of $\Phi^{4}$ model parameters for $\mathrm{PbTiO}_{3}$ corresponds to a crossover of displacive and order-disorder behavior obtained in the scope of the $\Phi^{4}$ model. 
Interestingly, the depth and position of the minimum of the calculated static potential for the ferroelectric distortion are substantially different for the low-temperature tetragonal and hightemperature cubic phases. The correlation of the calculated double well potential depth and the temperature of the ferroelectric transition favors a mixed displacive (near the tetragonal phase) and order-disorder ( $\mathrm{n}$ - ar the cubic phase) nature of the phase transition in $\mathrm{PbTiO}_{3}$.

Most recently, we have carried out calculations to describe a pressure induced phase transition at zero temperature.[20] Initial results shown in Fig. 2 indicate the presence of a tricritical point, i.e. it shows that compressibility of a parameter equal to zero at a tricritical point. Based on calculations of the optimized geometry, we will obtain the dependence of the ferroelectric distortion and strain on applied hydrostatic pressure.

Among some of the results obtained, the critical pressure of the second order tetragonalcubic phase transition was determined (18.8 GPA) at zero temperature. The point of the phase transition was found in the calculations of the optimized geometry with respect to c/a and $\delta Z$ (ferroelectric displacement parameters); note, that in these studies the experimental ratio for displacement of oxygen octahedra $\left(\delta Z_{0}\right)$ and $T_{i}$ atoms $\left(\delta T_{i}\right)$, namely $\delta Z_{0}: \delta Z_{T i}=0.121: 0.04$ was used. Some results are shown in Fig. 3. As in our zero pressure studies, as a next step, similar calculations for independent displacements, $\delta Z_{\mathrm{Ti}}$ and $\delta Z_{0}$, will be performed to study pressure induced effects.

Finally, we note that resulcs of FLMTO total energy calculations show that structural parameters for the ferroelectric ground state and their dependence on pressure were determined in reasonable agreement with experiment. Hence, the results obtained may be considered as the basis for further first-principles studies of pressure, the effect of impurities and the role of anisotropic tension which obviously plays an important role in the growth of monocrystalline films that are well known to be interesting from the technological point of view.

\section{C. $\mathrm{VO}_{2}$ and $\mathrm{TIO}_{2}$ Surfaces, Interfaces and Superlattices}

1. Eree TiO2surface. As a first important step in investigating ceramic-ceramic interfaces, we have end out highly precise FLAPW studies of the clean relaxed $\mathrm{TiO}_{2}(110)$ surface.[21,22] To our $b^{2}$, no first-principles results for the electronic structure and geometry optimization of $\mathrm{TiO}_{2}$ are available which were calculated by up-to-date precise techniques. The present paper comprises some of our most important results for the electronic structure and relaxed geometry for a clean $\mathrm{TiO}_{2}$ rutile (110) surface. The rutile (110) surface was chosen because it is the most stable single crystal surface for $\mathrm{TiO}_{2}$ and in addition has rather high symmetry which facilitates the calculation. 


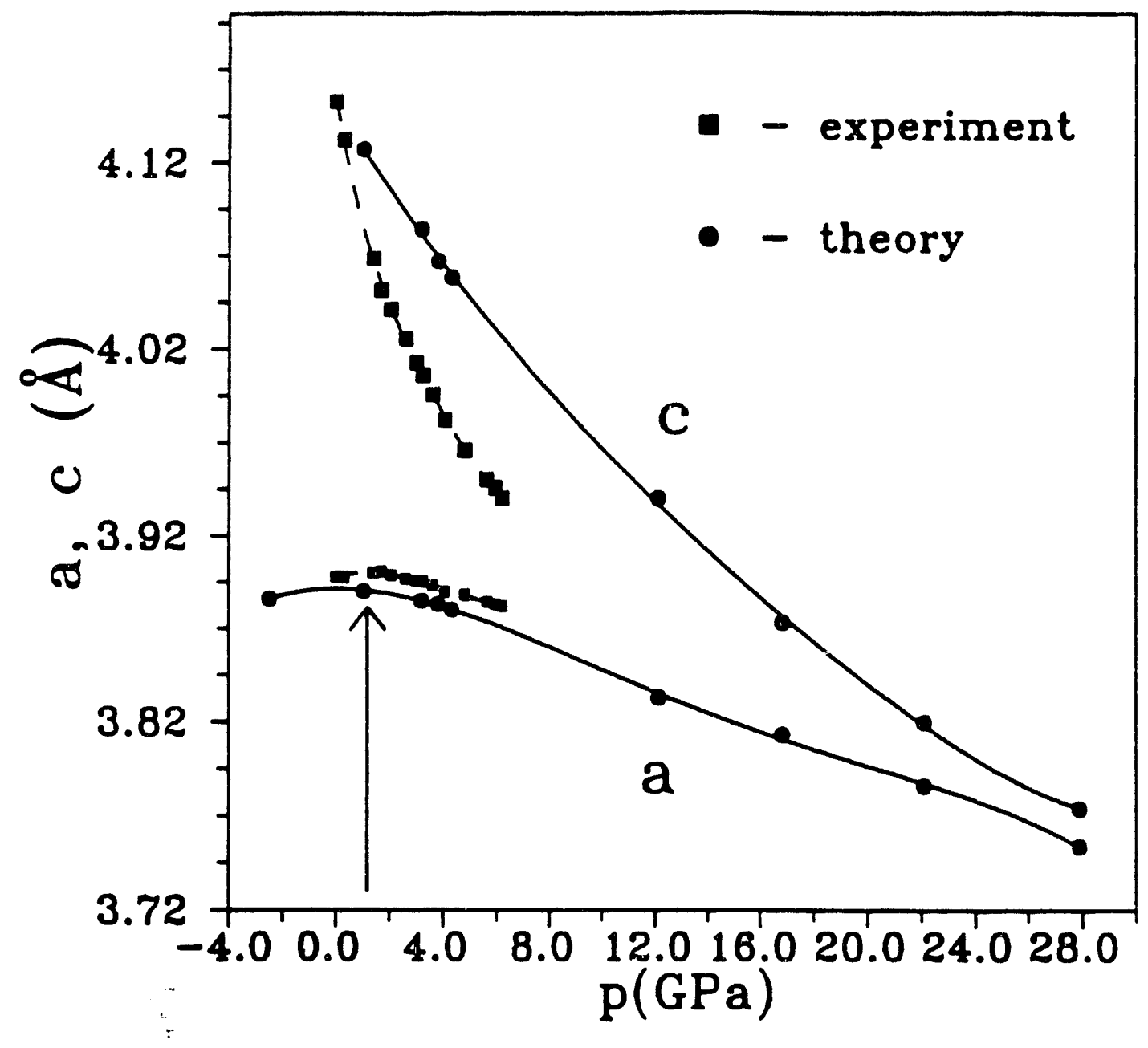

Figure 2. The pressure dependence of the a and $c(\AA)$ latice parameters of terragonal $\mathrm{PbTiO}_{3}$. 

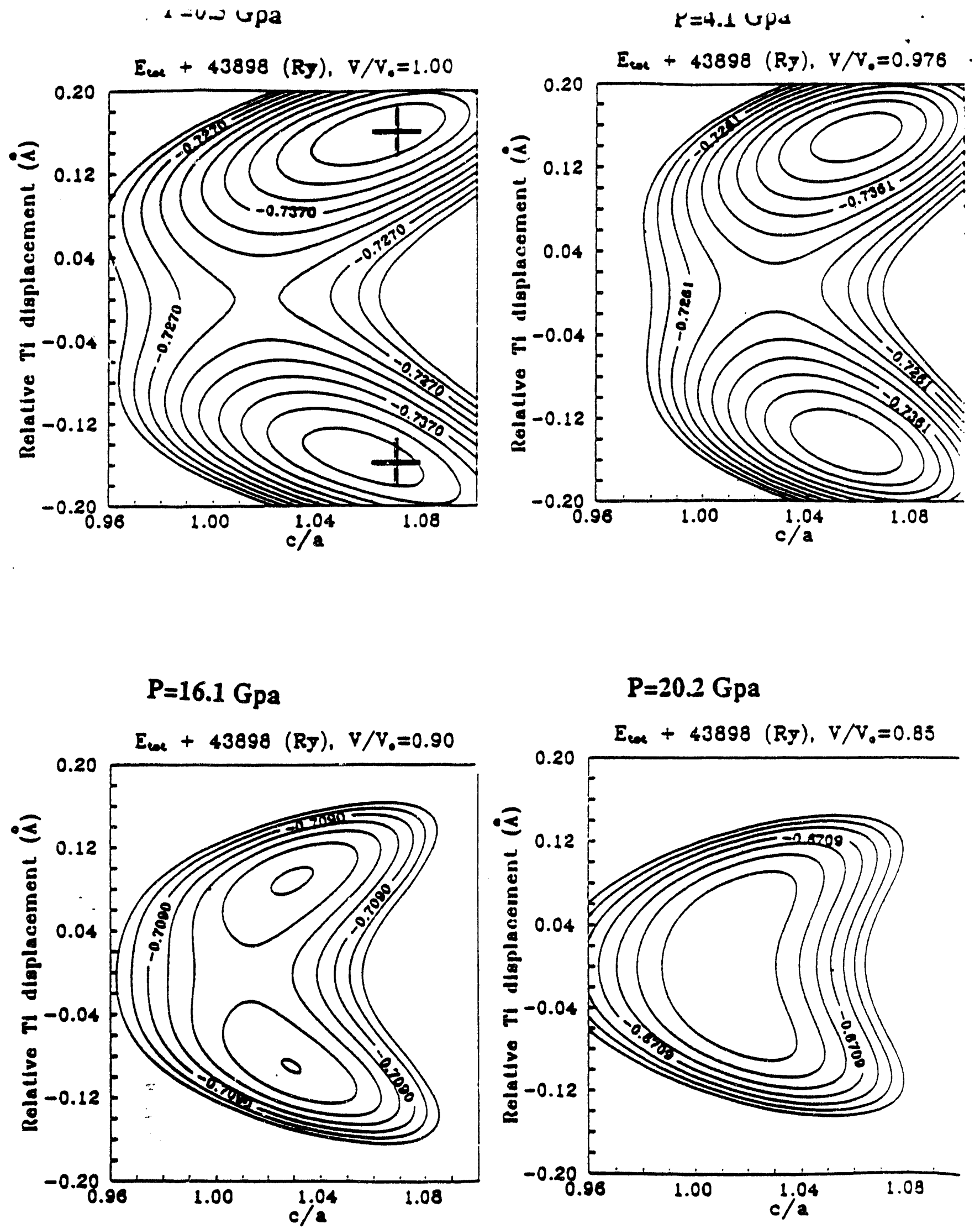

Figure 3. The potential energy surface contours for different pressures. Experimental structural parameters for the ferroelectric ground state are shown by crosses. 
The electronic structure and total energy were calculated by the self-consistent FLAPW method within the local-density approximation (LDA). In particular, we used the LDA exchangecorrelation potential of Hedin and Lundqvist during the self-consistency cycle and for geometry relaxation; four special $\mathbf{k}$-points were taken for the valence states, whereas one $\mathbf{k}$-point was chosen for a separate energy window to include Ti $3 s$ - and $3 p$-like states. The plane wave expansion of density and potential was cut off at $7.5 \mathrm{a} . \mathrm{u} .{ }^{-1}$, and the number of basis functions was restricted by a maximum momentum of 3.6 a.u. ${ }^{-1}$, resulting in $\sim 1300$ basis functions.

Surface states were found in the density of states, such as an s-like surface state at $-15 \mathrm{eV}$. Band bending states of width 0.5 appear just below the Fermi energy, in agreement with photoemission experiments. The positions of the atoms in the surface and subsurface layers and the corresponding change of Ti-O bond-lengths were derived by total energy minimization. In general, downward relaxations were obtained for which the 5-fold coordinated Ti experienced the largest relaxation of $-0.180 \AA$, where the second most important relaxation effect, $-0.156 \AA$, occurred for the surface $O$. The calculated Ti-O bond lengths are in very good agreement with experimental data for the $\mathrm{TiO}_{2}$ (100) surface. The calculated work function, $6.90 \mathrm{eV}$, compares favorably with the experimental result of $6.83 \mathrm{eV}$. Based on an extension of density functional theory by Fritsche and $\mathrm{Gu}$ (to be published) to treat excited states, the valence and conduction band gap was calculated to be $1.99 \mathrm{eV}$, which is in much better agreement with the experimental gap of $2.6 \mathrm{eV}$ when compared to the one-particle band gap of $0.65 \mathrm{eV}$.

2. YOz and TiO2 thin films and multilayers. We have completed the first phase of our work on the $\mathrm{VO}_{2}$ and $\mathrm{TiO}_{2}$ thin films and multilayers in collaboration with Dan Lam's group at Argonne National Laboratory. Electronic structure calculations [2] for $\mathrm{VO}_{2}$ and $\mathrm{TiO}_{2}$ bulk crystals in rutile structure, their surfaces and $\mathrm{VO}_{2} / \mathrm{TiO}_{2}$ superlattices were carried out by the FLMTO method and compared with experimental results obtained recently by Lam, et al., on epitaxial films.[1] Although the nature of surface states for $\mathrm{TiO}_{2}$ and $\mathrm{VO}_{2}$ appear to be very different, in the multilayered structures a common $\mathrm{VO}_{2}-\mathrm{TiO}_{2}$ conduction band was readily formed and the distinction between "insulating" $\mathrm{TiO}_{2}$ and "metallic" $\mathrm{VO}_{2}$ layers was almost lost in very thin multilayerud structures. Vanadium electrons are essentially delocalized into the $\mathrm{TiO}_{2}$ layers and the electronic eharge distributions in the $\mathrm{VO}_{2} / \mathrm{TiO}_{2}$ superlattice are similar to those in the usual $\mathrm{VO}_{2}$ rutile latuid. The results obtained gave some insight into the experimental data obtained by MOCVD for epitaxially grown VO/ $/ \mathrm{TiO}_{2}$ multilayers by Lam, et al.[1]

In order to go beyond these first calculations and study the real stucture observed by Lam et al., we need to investigate the (110) orientation of the multilayer structure system where the (110) face of rutile $\mathrm{TiO}_{2}$ is matched onto the (001) face of monoclinic $\mathrm{VO}_{2}$, which is known to be a non-magnetic semiconductor. As a first step, we have studied the metal-insulator transition at 333 $\mathrm{K}$ in bulk $\mathrm{VO}_{2}$ by calculating the electronic structure of its monoclinic phase. Surprisingly, we find 
that it is predicted to be a semi-metal - not the semiconductor found experimentally. As described below, and as a severe test of this failure of the LDA, we performed total energy FLMTO simulations by proceeding in steps from the rutile phase to the monoclinic phase. Again we find no energy gap.[23]

At this point, we need to note that there are still controversies on what is the driving force for this phase transition. Several points of view can be found currently in the literature.[24] First, the driving force is thought to be a crystal field which changes its symmetry in the process of monoclinic distortion of the rutile crystal and which leads to the splitting of $V 3 d$ states. The second says that this transition is of the Mott-Hubbard type. Whether the insulating phase of $\mathrm{VO}_{2}$ should be called a Mott insulator is a matter of debate. Since unlike most other possible Mott insulators for $\mathrm{VO}_{2}$, there is no sign of antiferromagnetic order. As alluded to above, our recent calculation[23] of the total energy of $\mathrm{VO}_{2}$ shows it to undergo a smooth transition from the rutile to the monoclinic structure which causes no change in volume but significant internal atomic rearrangement characterized as a dimerization of vanadium atoms along the $c$-axis. We find that there is no energy minimum along the distortion vectors and that no band gap opens for any distortion up to $30 \%$ larger than experimentally observed. Hence, we rule out that the conveational LDA approach, which usually describes good chemical (crystal) splitting of bands but does not treat correctly electronic correlations.

We believe that the driving force for the metal-insulator transition is a competition between "chemical" and "correlation" forces. As described in the next section, in order to shed some light on this, we propose (1) to improve the LDA scheme of calculations by including an additional Hubbard correlation term in the potential $\left(U_{H}\right)$ and (2) to implement this scheme in the FLMTO method. Based on this approach we hope to get the correct band structure and correct total energy behavior for the bulk metal-insulator transition. Once we have a reliable band structure for monoclinic $\mathrm{VO}_{2}$, we can proceed with more sophisticated models to investigate $\mathrm{TiO}_{2} / \mathrm{VO}_{2}$ interfaces and, in particular, the nature of the states at the interface, possible metallization, etc. 


\section{REFERENCES}

1. H.L.M. Chang, H. You, J.C. Parker, J. Guo, Y. Gao and D.J. Lam, in High Performance Ceramic Films and Coatings, ed. by P. Vincenzini, Elsevier, 1991, p. 161; H.L.M. Chang, H. You, J. Guo and D.J. Lam, Appl. Surf. Sci. 48/49 (1991) 12-18; R.P. Chiarello, H. You, H.L.M. Chang, T.J. Zhang and D.J. Lam, "The final size effect on the metal-insulator transition of MOCVD grown $\mathrm{VO}_{2}$ films", (preprint).

2. D.L. Novikov, V.A. Gubanov, A.J. Freeman, D.J. Lam, and M. Methfessel, "Electronic Structure of $\mathrm{VO}_{2}$ and $\mathrm{TiO}_{2}$ Thin Films and Multilayers", Phys. Rev. B (to appear).

3. E. Wimmer, H. : "rakauer, M. Weinert and A.J. Freeman, Phys. Rev. B 24, 864 (1981); M. Weinert, E. Wimmer and A.J. Freeman, Phys. Rev. B 26, 4571 (1982).

4. M. Springborg and O.K. Andersen, J. Chem. Phys. 87, 7125 (1987); M. Methfessel, Phys. Rev. B 38, 1537 (1988); M. Methfessel, C.O. Rodriguz and O.K. Andersen, Phys. Rev. B 40, 2009 (1989); A.T. Paxton, M. Methfessel and H. Polatoglu, Phys. Rev. B 42, 8127 (1990).

5. Chun Li, C.L. Fu and A.J. Freeman, Phys. Rev. B 48, 8137 (1993).

6. Chun Li and A.J. Freeman, Phys. Rev. B43, 780 (1991).

7. Y.Y. Huang, C. Liu and G.P. Felcher, Phys. Rev. B 47, 183 (1993).

8. R. Wu and A.J. Freeman, (in final preparation).

9. H. Jang, D.N. Seidman and K.L. Merkle, Scripta Metallurgica, 26, 1493 (1992).

10. J.A. Eastman and M. Rühle, Ceram.Eng.Sci.Proc. 10, 1515 (1989).

11. K.T. Park and A.J. Freeman, "Electronic Structure of $\mathrm{Pd} / \mathrm{Al}_{2} \mathrm{O}_{3}$ Interface", Phys. Rev. B (to appear).

12. K.T. Park, D.L. Novikov, V.A. Gubanov and A.J. Freeman, "Electronic Structure of Nobel-Metal Monoxides: PdO, PtO and AgO", Phys. Rev. B (submitted).

13. O. Mryasov, D.L. Novikov, K.T. Park, Q.W. Li and A.J. Freeman, "Electronic Structure, Optimal Geometry, Static Potential and Ferroelectric Instability in $\mathrm{PbTiO}_{3}$ ", Phys. Rev. B (submitued).

14. R.I schen, Nature, 358, 136 (1992).

15. R.Futzohen and H. Krakauer, Ferroelectrics, 136, 65 (1992).

16. A. Bussmann-Holder, Ferroelectrics, 136, 27 (1992) and references therein.

17. S. Padlewski, A.K. Evans, C. Ayling and V. Hein, J. Phys. C: Condensed Matter, 4, 4895 (1992).

18. V. Heine, X. Chen, S. Dattagupta, M.T. Dove, A. Evans, A.P. Giddy, S. Marais, S. Padlewski, E. Salje and F.S. Tautz, Ferroelectrics, 126, 255 (1992). 
19. E.K.H. Salje and S. Marais, Ferroelectrics, 136, 1 (1992).

20. O. Mryasov, D.L. Novikov, A.J. Freeman, "Pressure Induced Phase Transition in $\mathrm{PbTiO}_{3}$, (in preparation).

21. R. Podloucky, S.G. Steinemann and A.J. Freeman, J. Mat. Res. 2562 (1992) 43-48; R. Podloucky, S.G. Steinemann and A.J. Freeman, New J. Chem. 16, No. 12 (1992) 1139 1143.

22. D. Vogtenhuber, R. Podloucky, A. Neckel, S.G. Steinmann and A.J. Freeman, "Electronic Structure and Relaxed Geometry of the TiO2 Rutile (110) Surface", Phys. Rev. B (to appear).

23. O. Mryasov, D.L. Novikov and A.J. Freeman, "Failure of Local Density Theory for the Monoclinic Phase of $\mathrm{VO}_{2} "$, (in preparation). 


\section{A.J. Freeman \\ Publications Supported by the \\ U.S. DEPARTMENT OF ENERGY (DE-FG02-88ER45372) \\ 1990-present}

\section{Publications}

1. "Energetics, Bonding Mechanism and Electronic Structure of Metal/Ceramic Interfaces", (with Chun Li and C.L. Fu), Proc. Acta/Script Conf., Santa Barbara, CA, Jan. 1989, Metal-Ceramic Interfaces, eds. M. Rühle, A.G. Evans, M.F. Ashby, and J.P. Hirth, Pergamon Press (1990) p. 2.

2. "Structural, Electronic, and Magnetic Properties of Thin Films and Superlattices", (with A. Continenza and Chun Li), MRS Bull. Vol. XV.9, (1990) p. 27.

3. "Energetics, Bonding Mechanism and Electronic Structure of Meta/Ceramic Interfaces: $\mathrm{Ag} / \mathrm{MgO}(001) "$ ", (with Chun Li and C.L. Fu), Acta Scripta Conf., Santa Barbara, CA, Jan. 16-18, 1989; in Metal-Ceramic Interfaces, Proceedings of an International Workshop, eds. M. Rühle, A.G. Evans, M.F. Ashby, J.P. Hirth, Pergamon Press (1990), p. 2.

4. "Structural, Electronic, and Magnetic Properties of Interfaces", (with A. Continenza and Chun Li), to appear in Atomic-Level Properties of Interface Materials, ed. by D. Wolf and S. Yip, Chapman and Hall, Press, London, lst ed. 1991).

5. "Giant Monolayer Magnetization of Fe on MgO: A Nearly Ideal 2D Magnetic System", (with Chun Li), Phys. Rev. B 43, 780 (1991).

6. "Computational/Theoretical Approach to the Design of New Materials", (with S.P. Tang, A. Continenza, and C. Li), in Computer Aided Innovation of New Materials, M. Doyama, T. Suzuki, J. Kihara and R. Yamamoto, eds., (Elsevier Sci. publ. B.V., North-Holland, 1991), p. 143-153.

7. "Atomistic Study of the Clean and Hydroxylated TiO2 Surface", (with R. Podloucky and S.G. Steinemann), Mat. Res. Soc. Symp. Proc., 252 (1992) 43-48.

8. "Structural, Electronic and Magnetic Properties of Thin Films and Superlattices", (with A. Continenza and Chun Li), in Materials Interfaces: Atomic-Level Structure and Properties, D. Wolf and S. Yip, eds., Chapman and Hall Publ. (1992), pp. 275-298.

9. "First-Principles Calculations for the Clean and Hydrogenated TiO2 Rutile (110) Surface", (wittr. Podloucky and S.G. Steinemann), New. J. Chem. (1992) 16, 1139-1143.

10. "Encretics, Bonding Mechanism and Electronic Structure of Metal/Ceramic Interfaces: Ag/MgO(001)", (with C. Li and C.L. Fu), Phys.Rev. B 48, 8317 (1993).

11. "Electronic Structure of $\mathrm{Pd} / \mathrm{Al}_{2} \mathrm{O}_{3}$ Interface", (with K.T. Park) (to be published).

12. "Effects of Ternary Additions on the Twin Energy and Site Preference in $\gamma$-TiAl", (with Jian-hua Xu), J. Matls. Res. (submitted).

13. "Electronic Structure and Metal-Ceramic Bonding of the $\mathrm{Al}_{2} \mathrm{O}_{3}$-Pd Interface", (with KeyTaeck Park), Phys. Rev. B (submitted). 
14. "Electronic Structure of $\mathrm{VO}_{2}$ and $\mathrm{TiO}_{2}$ Thin Films and Multilayers", (with V.A. Gubanov, D.L. Novikov and D.J. Lam), Phys. Rev. B (submitted).

15. "Magnetism at Metal-Ceramic Interfaces: Effects of a Au Overlayer on the Magnetic Properties of Fe/MgO(001)", (with Ruqian Wu), J. Magn. Magn. Matls. (to appear).

16. "Electronic Structure of Noble-metal Monoxides: PdO, PtO, AgO", (with K.T. Park, D.L. Novikov and V.A. Gubanov), Phys.Rev. B (to appear).

17. Electronic Structure and Relaxed Geometry of the $\mathrm{TiO}_{2}$ rutile (110) surface", (with D. Vogtenhuber, R. Podloucky, A. Neckel and S.G. Steinemann), Phys. Rev. (to appear).

18. "Effect of Au Overlayer on the Magnetism and Properties of $\mathrm{Fe} / \mathrm{Mg}(001)$, (with $\mathrm{R}$. Wu), J. Magn. Magn. Matls. (to appear).

19. "Electronic Structure, Frozen Phonons and Origin of the Ferroelectricity Instability in $\mathrm{PbTiO}_{3}$ ", (with Guangwei Li and Key Taeck Park), (submitted).

20. "Optimized Geometry, Static Potential, Electronic Structure and Ferroelectric Instability in $\mathrm{PbTiO}_{3}$ ", (with O.N. Mryasov, D.L. Novikov, K.T. Park and G. Li), Phys. Rev. B (submitted).

\section{Presentations}

"Electronic Structure and Magnetism of Metal Surfaces, Overlayers and Interfaces", (with Chun Li and R.Q. Wu), Proc. NATO Summer School, Greece, June 1990.

"Structural, Electronic and Magnetic Properties of Metal/Ceramic Interfaces: Fe/MgO(001)" (with Chun), paper presented at the March 1990 APS Mtg., Anaheim, CA.

"Computational/Theoretical Approach to the Design of New Materials", (with S.P. Tang, A. Continenza, and C. Li), invited paper for Proc. Intl. Conf. on Computer Applications to Matls. Sci. \& Engr., Tokyo, Japan, Aug. 28-31, 1990.

"Atomistic Study of the Clean and Hydroxylated TiO2 Surface", (with R. Podloucky and S.G. Steinemann), Fall Matls. Res. Soc. Mtg., Boston, MA, Dec. 2-6, 1991.

"Electronic Structure, Froeen Phonons and Origin of the Ferroelectricity Instability in PbTiO3", (with Gunnwei Li and Key Taeck Park), paper presented at APS Mtg., Indianapolis, IN, March 16-2t, 1992.

"Electronis Sucture of Thin Films and Multilayers of TiO2 and VO2" (with D.L. Novikov and V.A. Gubanov), paper presented at APS Mtg., Indianapolis, IN, March 16-22, 1992.

"Ceramic-Ceramic Interfaces in Epitaxially Grown $\mathrm{VO}_{2} / \mathrm{TiO}_{2}$ Multilayers: Full-Potential LMTO Investigations", (with D.L. Novikov and V.A. Gubanov), paper presented at the 1993 March Meeting of The American Physical Society, Seattle, Washington, March 22-26, 1993.

"Electronic Properties of $\alpha-\mathrm{Al}_{2} \mathrm{O}_{3}$ and the $\mathrm{Al}_{2} \mathrm{O}_{3}$-Pd Interface", (with Key-Taeck Park), paper presented at the 1993 March Meeting of The American Physical Society, Seattle, Washington, March 22-26, 1993. 
"Local Spin-density Theory of Interface and Surface Magnetocrystalline Anisotropy", (with Ding-sheng Wang and Ruqian Wu), invited talk for the 38th Annual Conference on Magnetism and Magnetic Materials, Minneapolis, MN, November 15-18, 1993.

"First Principles Determination of X-ray Magnetic Circular Dichroism and Validity of its Sum Rules for Spin and Orbital Angular Momentum" (with Ruqian Wu), invited talk for the 38th Annual Conference on Magnetism and Magnetic Materials, Minneapolis, MN, November 15$18,1993$.

"Electronic Properties of a- $\mathrm{Al}_{2} \mathrm{O}_{3}$ and the $\mathrm{Al}_{2} \mathrm{O}_{3}$-Pd Interface", (with Key-Taeck Park), paper to be presented at the 1994 March Meeting of The American Physical Society, Pittsburgh, PA, March 21-25, 1994.

"Ceramic-Ceramic Interfaces in Epitaxially Grown $\mathrm{VO}_{2} / \mathrm{TiO}_{2}$ Multilayers: Full-potential LMTO Investigavions", (with D.L. Novikov and V.A. Gubnanov), paper to be presented at the 1994 March Meeting of The American Physical Society, Pittsburgh, PA, March 21-25, 1994. 

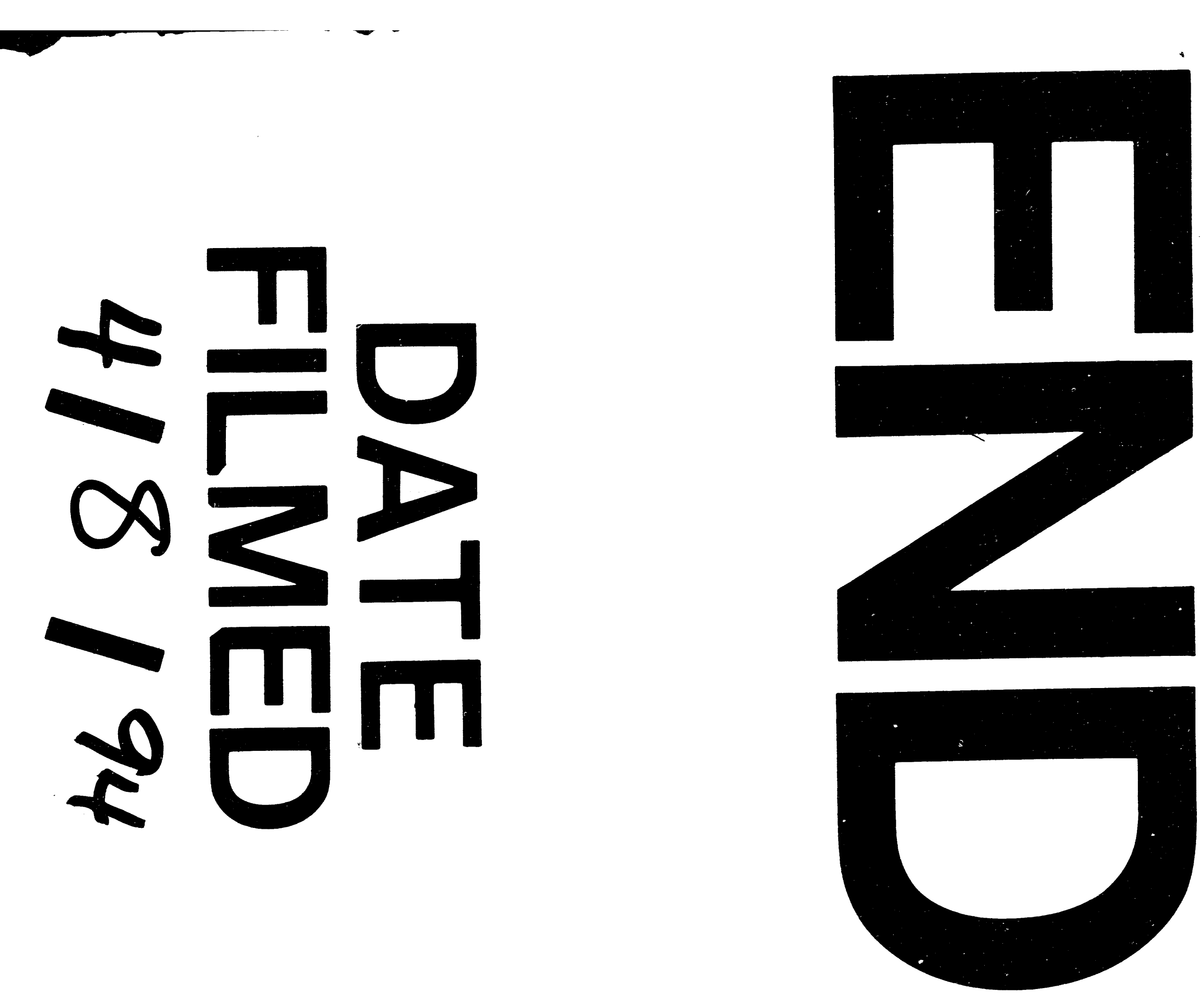
